\title{
Urgences
}

\section{Lettre sur le sujet de la passion ou l'invention du lecteur}

\section{Anne Élaine Cliche}

Numéro 30, décembre 1990

L'autre du texte

URI : https://id.erudit.org/iderudit/025626ar

DOI : https://doi.org/10.7202/025626ar

Aller au sommaire du numéro

Éditeur(s)

Urgences

ISSN

0226-9554 (imprimé)

1927-3924 (numérique)

Découvrir la revue

Citer cet article

Cliche, A. É. (1990). Lettre sur le sujet de la passion ou l'invention du lecteur.

Urgences, (30), 58-69. https://doi.org/10.7202/025626ar d'utilisation que vous pouvez consulter en ligne.

https://apropos.erudit.org/fr/usagers/politique-dutilisation/ 


\title{
Lettre sur le sujet de la passion ou l'invention du lecteur \\ Anne Élaine Cliche
}

\author{
C'est d'amour qu'il s'agit, enfer et paradis, et \\ uniquement de ça
}

Jean-Luc Godard, à propos de Passion

Dante marche avec Sade dans l'Enfer de la Bibliothèque.

- Mon cher Sade, sourit Dante, il me semble que l'on vous lit de plus en plus. N'êtes-vous pas étonné ou troublé tout au moins d'occuper aujourd'hui une place fort honorable parmi les vivants lecteurs?

- Certes, on me lit, mon ami, on me lit, répond Sade en ralentissant le pas. Il est vrai que l'on parle beaucoup de moi par les temps qui courent. Mais votre question m'amène plutôt à vous dire que, vu d'ici, je ne sais plus très bien qui sont les vivants et qui sont les morts. Peut-être ces lecteurs dont vous me parlez sont-ils en effet bien vivants, trop vivants justement! Je veux dire: pour lire. Permettez-moi de préciser ma pensée: je cherche à rendre compte de l'effroyable sang-froid et j'irais jusqu'à dire, excusez-moi, de l'incroyable frigidité avec laquelle ils nous abordent ici, dans le texte. Depuis que la psychanalyse a fait de moi un adjectif, on me prend davantage pour un cas clinique inégalé ou pour le nourrisson universel, rarement pour un dramatique humoriste, moi qui n'ai jamais cessé de me prendre pour tel. À vous, mon cher Dante, je demanderai: y a-t-il seulement un lecteur amoureux? Car enfin, vous êtes un des rares à connaître la part de passion et de sacrifice qu'implique la rencontre avec la parole des morts. (Ici, Sade baisse un peu la voix.) Et le texte n'est-il pas le seul lieu où les morts parlent et où les parlants meurent?

(On entend le rire de Dante.)

Tenez, à propos, reprend Sade, je désire vous exposer quelque chose dont vous savez tout mais qu'il me plait de faire résonner ici, et c'est que mon œuvre - tout ce que j'ai écrit - n'a été somme toute qu'un accomplissement littéral de la Passion, entendez: la rencontre, ou mieux (rire), la crucifiction de l'amour et de la souffrance. Ce ne sont là que 
"les crimes de l'amour". Et ma Société des Amis du Crime avec tout ce qu'elle a mis en œuvre ne peut se lire, et se lire intégralement, que comme une Imitation de Jésus Christ (rires). Disons plutôt une physique de l'Tmitation.

Sade s'est arrêté. Il prend le bras de Dante:

- Or, à ce titre, dit-il encore, vous reconnaîtrez avec moi que le lecteur est rare. Finalement, très peu parviennent à ce que vous-même avez appelé le sens anagogique du texte, et que je ne saurais nommer pour ma part que le «supplice intégral du lecteur* ou, si vous préférez, son Incarnation. Mais laissons cela, Maître, ajoute enfin Sade en reprenant le pas, et dites-moi plutôt ce qu'il en est de vous.

- Oh, quant à mes lecteurs, ils s'affairent, ils s'affairent, mais nous verrons cela plus tard car pour le moment je vous écoute attentivement, Marquis, et mieux: je vous entends, répond le Maître ému. Comme toujours, d'ailleurs, je vous prends à la lettre. Ce qui a pour effet de me conduire directement au sens supérieur. Effet non négligeable. Et puis, autant vous avouer ce que vous savez déjà (et que vous êtes peut-être le seul à savoir): je ne cesse de vous relire en tremblant et en pleurant. La passion du crime dont vous consumez vos écrits n'est pas loin de ce dont je me suis fait, moi, le corps d'écriture. Comment dire.... il me semble que votre "physique" de l'Imitation rejoint la mienne en ce point précis où le corps devient le signifiant brut de sa lecture. Figurezvous que récemment, il m'est apparu en rêve que vous faisiez une magistrale mise en scène de ma Comédie. Et par je ne sais quelle étrangeté, il se dégageait de tout cela une impression troublante de familiarité, de déjà-vu. Voilà, je songeais que nous pourrions travailler à la réalisation de ce rêve. Cela s'appellerait *Dante avec Sade», ou encore, * Dante selon Sade». Qu'en pensez vous?

Une ombre qui les accompagne depuis un moment fait alors entendre un grand rire. Sade s'efface pour laisser passer nul autre que saint Hubert Aquin. (Dans l'Enfer de la Bibliothèque, la sainteté renvoie, on s'en doute, à l'envers ou à l'enfer - du sacré, c'est à -dire à ses « dessous *.)

- Vous semblez oublier, dit celui-ci en s'avançant, que j'ai moi-même tenté à plusieurs reprises d'écrire cette mise en scène. Mes titres différaient du vôtre mais il est facile de voir qu'il s'agit du même projet. De là, si vous permettez, l'étrange familiarité de votre rêve, mon Seigneur. 
60

- Il faudrait imaginer cette fresque inconnue d'un Dante rêvant les livres d'Hubert Aquin, s'empresse d'ajouter le Marquis amusé.

- Je serais ravi de reprendre tout cela avec vous, poursuit Aquin. L'effet est assuré. Effet que j'avais autrefois nommé «l'invention de la mort». Mort du lecteur, bien entendu.

Sade s'approche:

- Et dis-moi, mon frère, où donc a lieu cet effet que tu appelles d'un si beau nom?

- Dans la lettre, D.A.F., dans la lettre. Tu ne lignores pas.

Sade s'incline:

- On commence?

Arrêtons-nous ici, au moment où Dante, Sade et Aquin s'apprêtent à relire La divine comédie, pour reprendre à notre compte cette pensée qu'ils énoncent d'une "passion dans la lettre . Passion dans la lettre que je voudrais pour ma part mettre en scène dans cet écrit que je vous destine. Je vous écris, dirais-je, pour n'être pas tout à fait là où je parle mais bien plutôt là d'où vous me lisez. Où cela? demanderez-vous, s'il vous vient à l'esprit que tout ceci n'est que supercherie et sophisme d'énonciation. Où cela? Eh bien précisément à la place où cette question surgit. Cette place, la vôtre, est justement ce dont je veux vous entretenir, en cela surtout qu'elle implique plus qu'une action, soit: une passion, comme une passation, entendez: la transmission d'un désir en souffrance. Place d'un corps déictique qui n'est celui de personne et qui pourtant se marque d'un singulier. Disons: le corps de «quiconque * prenant à revers son étymologie. Ce qui qu'oncques (qui... jamais) est le corps qui lit et qui n'est jamais que ce corps-là. Non pas un corps sans nom, sans lettre mais un corps pris à la lettre et dans la lettre; le corps, enfin, de la lettre. Corps de quiconque vient là pour accomplir l'écriture.

Après une telle disposition narrative, je peux dire plus simplement ce qui s'entend déjà, peut-être, dans les premiers mots de cet envoi, à savoir que lire constitue l'acte même de la passion. Je commencerai donc ainsi: 


\section{Chers lecteurs,}

Dans le tableau du Christ en croix que peint le Bossuet du Sermon sur la Passion - tableau remarquable par sa violence spectaculaire et baroque - , dans ce tableau, donc, les cris, les exhortations, les questions, les prières se superposent et semblent provenir d'une triple énonciation: celle de l'auteur du Sermon, celle des fidèles qui l'écoutent lire et celle-là même des acteurs de cette Passion (le Christ, Marie, la foule, etc.). Le rythme du tableau est aussi scandé par un regard atterré tendu vers l'Agneau sanglant. Bref, il y a là toute une dispositio du pâtir et du compatir, mais surtout il y a là un verbe (la langue de Bossuet) dont la littéralité se construit pour aboutir à une formulation finale presque comique où l'on découvre que ce qui s'offre à la contemplation n'est rien d'autre que la figure d'un lecteur. Figure d'un Christ lecteur assidu et acteur non moins "consommé * des Écritures. Figure accomplie, dirais-je aussi, du comédien. Voici: à la fin du Sermon, après une description minutieuse de l'angoisse et de la culpabilité du Christ - «jusque dans la moëlle des os » dit le psaume -, Bossuet écrit:

Je pourrais ici, Chrétiens, vous faire une vive peinture d'un Jésus mourant et agonisant, défaillant peu à peu, attirant l'air avec peine d'une bouche toujours ouverte et livide et trainant lentement les derniers soupirs par une respiration languissante, jusqu'à ce qu'enfin l'âme se retire et laisse le corps froid et immobile. Ce récit pourrait peut-être émouvoir vos cœurs; mais il ne faut pas travailler à vous attendrir par de vaines imaginations. Jésus n'est pas mort de la sorte. II fait l'un après l'autre ce qu'il a à faire: il parcourt toutes les prophéties pour voir s'il reste encore quelque chose [...] puis élevant sa voix avec un grand cri [...] il dit hautement: a tout est consommén.

Relisez cette phrase: «il parcourt toutes les prophéties pour voir s'il reste encore quelque chose *. Elle résume ici, dans une espèce d'au-delà du pathos où le texte accède d'un seul coup, toute la Passion d'un Christ-lecteur en tant que son acte d'accomplissement consiste uniquement - pour reprendre une formule de Lacan - à "pâtir du signifiant *. Qu'est-ce à dire? Ceci, peut-être, que le saut qualitatif opéré par le texte de Bossuet nous rappelle que l'expérience radicale de l'Écriture conduit celui qui la fait à la place intenable où se nouent l'action de l'Auteur (celui qui dicte ou 
62

rédige les Ecritures) et la passion du lecteur (ici: le Christ), dans le temps où cette action rencontre aussi sa cause, à savoir la Passion d venir de l'acteur.

Car c'est de lui, l'acteur, qu'il est déjà question dans le rouleau du Livre de l'Aricien Testament, c'est lui qui, déjà, y est mis en scène, et tout le Nouveau Testament ne s'écrit à son tour qu'en perpétuelle citation de l'autre texte. Le sens de la Passion du Christ n'aura été lisible - en son futur antérieur - que par la place qu'elle occupe dans le réarrangement des signifiants bibliques et théologiques. Or, il s'agit bien là d'une place intenable puisqu'en son point d'ombilic * tout est consommé ». Il n'y a plus alors, dit Bossuet, qu'une " mort à consomption".

De là, je peux dire que la logique catholique ne me retient que parce qu'elle est avant tout une logique de la lecture. La trinité acteur-auteur-lecteur se révèle finalement être l'équation d'un nouage dont le corps mourant est le signifiant brut: le signifiant d'un lieu vide à l'image du tombeau ouvert, la place circonscrite d'une consumation et d'une éclipse ou, si l'on veut, d'un rapt qui signe ici l'accomplissement. Ce qui est accompli ou consommé c'est le corps de cette lecture, le corps de sa comédie. Car la Passion est bien la comédie d'une lecture qui prend acte, à la lettre, de l'Écriture pour en exécuter non pas le sens - le sens n'est nullement épuisé par la Croix et semble plutôt reparcouru dans tous les sens; * une folie ", dit saint Paul -, mais pour exécuter, mettre'à exécution, le foyer d'énonciation.

Le comique de l'histoire n'est pas dans l'économie de la souffrance et de la culpabilité mais dans ce que cette économie vient dévoiler, à savoir l'oubli de Dieu, sa disparition, son néant. Le comique, dit Lacan, c'est le phallus qui s'allait dévoiler. Depuis le Mont des Oliviers jusqu'à la Croix, le Christ porte réellement toutes les fautes de l'humanité. Mais ce « réellement * réside uniquement dans l'expérience du langage qui s'expie puisqu'il s'agit de maintenir les Écritures à la hauteur d'une lecture « mortelle». Le scripteur et le lecteur, irréductibles l'un à l'autre, sont reportés au même dehors. * Jouer la comédie, dit François Régnault dans Le spectateur, c'est assumer sa propre causalité quand la cause est visiblement ailleurs que dans le masque qui vous définit". Autrement dit, la Passion est une comédie - et c'est en cela seulement qu'elle dit la vérité - en ce que sa cause réside dans 
l'au-delà et l'en-deça de la divinité du Christ (la divinité vient à manquer... de tout temps). Causalité soustractive que l'accomplissement dévoile dans son arbitraire le plus essentiel. L'action de l'Auteur a pour cible celui qui est Lui (Dieu) et pourtant autre que Lui (le Christ). Elle a pour cible la passion d'un lecteur.

Bataille: «La mort de Jésus participe de la comédie dans la mesure où l'on ne saurait sans arbitraire introduire l'oubli de sa divinité éternelle - qui lui appartient - dans la conscience d'un Dieu tout-puissant et infini. .

Accomplir l'écriture, au fond, ce n'est rien d'autre que cela: lire pour actualiser l'épreuve mortelle de la lettre - son " arbitraire * - selon une corporalité triple et une: acteurauteur-lecteur. Ce corps actuel de la lecture nous ramène un moment à Dante dont la conversation avec Sade et Aquin a pris un tour un peu plus sophistiqué. N'oublions pas que l'Enfer de la Bibliothèque est le lieu où la philosophie la plus haute côtoie avec profit les pensées les plus scabreuses.

\section{$* * *$}

Dante, Sade et Aquin sont assis sur la scène qui se trouve au centre de l'Enfer de la Bibliothèque.

- Cher Marquis, souffle Dante, nos corps sont brisés après ce premier acte dans lequel vous ne nous avez rien épargné. Les opérations que vous faites subir aux corps sont une véritable matérialisation de la prière. Je propose un temps d'arrêt pour vous exposer quelques-unes de mes plus chères réflexions.

- Puis-je recueillir vos paroles? demande vivement Aquin dont le magnétophone est déjà en train d'enregistrer la voix douce et chantante du maitre italien.

- Faites donc, mon ami, faites donc.

Le Marquis pose son texte et s'approche respectueusement du poète qui se lève:

- Nous vous écoutons. La bande tourne.

Dante inspire et commence:

- Voici. Vous me demandiez, Marquis, comment se portent mes lecteurs et je ne sais que vous répondre sinon qu'ils compulsent à mon égard. Mais j'aimerais dire ceci qui 
64

vous intéressera davantage: il ne s'est jamais agi pour moi que de lecture. Rien d'autre, rien d'autre. Entendez: il n'y a pas d'écriture, pas de sujet, pas d'auteur, pas d'acteur, pas de livre et j'ajouterai: pas d'amour sans au moins un lecteur. Dans la * forêt italique * - que vous avez fort bien rendue tout à l'heure dans votre montage éprouvant - , dans cette forêt du monde, dis-je, le premier homme, mon cher Sade, est un lecteur. Et c'est parce qu'il lit, uniquement parce que tout à coup - ça lui tombe dessus - il lit, qu'il va pâtir, aimer et mourir. Souvenez-vous des premiers mots de ma Vita nova.

Aquin se lève pour lire de sa voix un peu traînante la phrase qui ouvre le « petit livre $\star$ :

Dans cette partie du livre de ma mémoire avant laquelle il y avait peu à lire, se trouve un titre, qui dit ceci: Incipit vita nova .

Dante lève la main pour l'interrompre:

- Merci, chère âme, vous me touchez jusqu'aux larmes et j'ai peine à poursuivre.

Sade dirige l'éclairage sur le visage troublé de Dante qui, debout, reprend le fil de son petit discours :

- La vie nouvelle commence là où il y a da lire et ce lieu est la mémoire, c'est-à-dire un non-lieu. Car la mémoire est un corps d'écriture et jamais un corps écrit. Cette mémoire, je la dirais corps prescrit à une lecture dans l'écriture. La Vita nova, comme vous savez, est l'écriture d'une naissance à l'écriture dont la fin est la condition de l'incipit. Rappelez-vous la vision innommée et innommable du dernier paragraphe. Eh bien, cette vision irregardable, si je puis dire, n'est pas l'aboutissement du livre mais son point de départ. C'est dire que la Vita nova ne procède pas selon un tâtonnement progressif d'énigme en énigme mais qu'elle est bien plutôt la mise en acte de l'énigme comme facteur d'une énonciation trinitaire: récitpoème-interprétation. L'énigme est là, donnée d'une seule voix.

Dante baigne à présent dans une lumière qui semble émaner de lui. Il va faire une révélation:

- Lecteur bien-aimé, * aiguise ici ton regard sur le vrai, car le voile à présent est si mince qu'il devient aisé à traverser». Écoute ceci: La Vita nova est écrit selon trois niveaux qui se recoupent et se répondent en des densités différentes pour laisser toujours échapper le sens. Car le sens est la 
ponctuation du désir de quiconque subit physiquement la révélation. Je suis l'acteur de tous mes livres, mais un acteur dont l'acte en est uniquement un de lecture. En effet, l'acte particulier du comédien exige de lui qu'il voit son langage, qu'il parcoure sa diction. Ainsi, doit-il éternellement transiter vers la position qui permette d'apercevoir ce langage, de filmer cette diction en train de s'écrire. Voilà, lecteur, ce que moi je te donne à lire et ce n'est rien d'autre qu'une circulation du mystère qui passe d'une densité à l'autre, selon une ponctuation qui relance le sens au lieu de l'arrêter, le faisant changer de lieu, de forme, de masse, mais lui conservant dans le texte son indissoluble opacité. La révélation est cette douleur hétérogène au texte, et elle en est la cause. (Ici, un court silence.) La mort de Béatrice n'est-elle pas le plus profond mystère et la souffrance même de ma langue qui a cherché à dire d'elle ce qui jamais ne fut dit d'aucune?

Aquin souffle quelque chose à l'oreille de Sade. Dante semble transfiguré par son texte:

- La passion est ma gnose et la gnose est ma Béatrice. Voilà ma seule hérésie. Mon texte entier est tourné vers ce lieu vide qui le lit et auquel je m'adresse ici encore pour faire assompter, là, cette deixis ou cette diction dans la langue. Et toi, lecteur, tu te demandes encore quelle est cette révélation sur laquelle je suis intarissable? Ne retiens que ceci: elle est l'interruption, l'arrêt intime de ta propre mémoire, qui te met en danger. Elle n'a de bénéfice qu'à représenter en figure de passage la place de ton corps à l'écrit.

La scène de l'Enfer est plongée dans le noir. Seul le corps de Dante irradie et semble transiter. La bande magnétique arrive à sa fin et quelques spectateurs infernaux se rassemblent devant le comédien fluorescent. Enfin, Sade se penche vers Dante:

- Maître, dit-il, vos propos me transportent car ils sont au plus près de désigner ce lieu secret et expérimental où s'opère chez moi la transformation des corps. Le mystère dont vous parlez, et qui n'est jamais que ce qui pointe le lecteur à sa place, relève aussi, n'est-ce pas, du bordel et du laboratoire. Ne sommes-nous pas au cœur d'un sacrifice?

Aquin s'est approché:

- Si vous permettez, j'aimerais que nous reprenions directement de là. 
66

Laissons à nouveau l'Enfer et son thêatre pour revenir à cette autre scène qu'est la scène de l'écriture. Je vous écris, ai-je dit. Et vous écrivant cette lettre sur la passion, je vous constitue pour un temps lecteurs.

Mais supposez un instant que cette lettre en soit une d'amour et qu'elle s'adresse, par sa charge érotique et passionnelle, à ce qui en vous ne peut en aucun cas appartenir à l'ensemble. Supposez que ma lettre soit à ce point intime que vous ne puissiez en supporter la lecture publique. Sa publication constituerait alors un viol, une mise à nu de ce qu'il y a de plus sacré en chaque sujet, à savoir l'ouverture, ou disons: le trou par où l'autre l'affole. Ce viol prescrit au regard qui lit, serait alors le moment où le désir s'actualise d'avoir à pâtir du signifiant. En d'autres termes, * pâtir du signifiant * par la lecture suppose que le texte soit pour le lecteur une question de vie ou de mort et que, le reconnaissant, ce lecteur accède au sacrifice de son être, à la mort de l'individu en lui, et s'offre au supplice d'avoir à transmettre l'écriture, c'est-à-dire à l'aimer. Souffrance d'autant plus grande, nous dit Aquin, que * le lecteur a tout fait pour être violé *.

Ainsi, l'acte de passion cherche son corps et c'est pour cela qu'il l'invente. *L'invention de la mort *, comme l'invention de la croix, consiste à trouver la place où clouer mon désir en souffrance. Et c'est là que Bossuet nous attend. En effet, ce désir, je peux le regarder mourir en de *vaines imaginations * et m'aveugler à sa souffrance car ce que je verrai alors ne sera que ma propre mort prise au miroir imparlable. À cela Bossuet oppose un crucifié en train de lire, un corps cloué à la dérive de la lettre et en train de parcourir minutieusement les prophéties qui l'annoncent, pour voir, justement, s'il n'y a pas un reste à cette théâtralité. Ce qui reste est alors cette division de l'écriture à la lecture dans laquelle le comédien n'a plus qu'à «assumer sa propre causalité », c'est-à-dire à embrayer son commencement réel sur le commencement fictif prévu par le texte.

La passion, dirais-je, ne va pas sans cette fiction, sans cette invention incroyable d'un verbe se faisant chair. Car de quelle invention s'agit-il dans la lettre - dans la lettre d'amour - sinon de l'invention de l'Autre, mais de l'Autre en tant qu'il rejoue pour moi l'effraction angoissante qui m'a fait corps, ou disons pour faire vite, en tant qu'il rejoue ma 
naissance et donc ma mort? Méconnaître cette fiction pour aller droit au corps de l'Autre conduit le pathos à la dévoration du signifiant. Ce corps à corps est aussi bien celui de la jalousie qui se leurre infiniment quant au lieu miroitant de son objet.

- La passion du moi dont témoigne le corps à corps, et qui peut mener à la mort réelle du sujet qui s'y livre, pourrait ici se penser comme un impouvoir de lecture. D'où ceci que la passion sans la lettre peut avoir pour effet qu'un sujet, si je puis dire, se trompe de mort et s'y plante. La passion doit passer par la lettre pour que l'impossible qui la destine soit symbolisé. «Pâtir du signifiant * serait alors souffrir que la lettre ou la langue d'amour pointe en la bordant la place par où ma mort se transmet. La lettre, c'est la frange de l'aimé(e), qui le met en creux ou en pointe et le rend infigurable au miroir. Que le sujet se précipite dans cette ouverture-éclair et c'est la mort, l'aliénation ou la psychose. La passion de lire s'en distingue de s'offrir au sujet comme la vérité de sa mort. Ce qui est autre chose que sa mort vraie.

* Quand un existant est au plus près d'être en même temps en deux endroits, dit Kierkegaard, il est passionné». Le corps est au supplice dans le temps où il perd ce qu'il trouve, temps où il fait le deuil de ce qu'il invente. Invention de la mort qui nous reprécipite dans l'Enfer de la Bibliothèque.

\section{***}

La lumière fluorescente dont le corps de Dante est devenu lè foyer d'émission enchâsse à présent toute la scène. Nous approchons de la fin d'une représentation de la Comédie et l'on commence seulement d'apercevoir la présence d'un appareillage compliqué encombrant l'avant-scène. Nous comprenons tout à coup que ce thêâtre est régi depuis le début par l'œil vitreux d'une caméra derrière laquelle - on le distingue bien maintenant - se tient Hubert Aquin. Ainsi, par l'entremise du saint scénariste, la nouvelle Comédie est diffusée dans tout l'Enfer sur écrans géants de télévision. Aquin s'approche de Sade, resté debout sur la scène, de façon à le filmer selon un morcellement impitoyable. Il s'adresse au Marquis:

- Dans l'Incarnation, dit-il, le rapport à la vue prédomine. La passion réveille toujours une hantise de la figurabilité. Cela s'explique, je crois, par le profond mystère 
68

de la Trinité irregardable et par le retour, en nous, d'une situation immémoriale où l'objet n'était pas signifié. L'image - la représentation - est ainsi immanquablement la faute originelle. Elle vient là où le foyer de symbolisation est mis hors circuit. Aujourd'hui, le monde semble entré dans un purgatoire de l'image. Moment où la faute tend à s'effacer à se donner même pour non avenue. Mais que devient l'image au Paradis? La vision impossible de la face de Dieu n'est peut-être que le retour dans le champ optique du foyer de symbolisation. Mentends-tu, D.A.F.?

- À merveille! D'ailleurs à propos d'entendre, j'ajouterai à tes savants développements que ce "foyer de symbolisation », comme tu l'appelles, n'est jamais que l'œil qui lit. Car l'œil qui lit ne voit pas. Il entend. Cent-vingt journées pour dire que le crime s'engendre à l'oreille!

Soudain, l'écran est envahi par le visage de Sade. La caméra parcourt cette célèbre face de pierre dont la divinité est accentuée par l'effet des rayons cathodiques. Arrêt brusque sur la bouche du Marquis. Les écrans de l'Enfer ne projettent plus que ses lèvres parlantes:

— * Il est reçu parmi les véritables libertins que les sensations comuniquées par l'organe de l'ouïe sont celles qui flattent davantage et dont les impressions sont les plus vives. En conséquence, les scélérats qui voulaient que la volupté s'imprégnât dans leur cœur aussi avant et aussi profondément qu'elle pouvait pénétrer, avaient imaginé une chose assez singulière. Il s'agissait, après s'être entouré de tout ce qui pouvait satisfaire les autres sens par la lubricité, de se faire en cette situation raconter avec le plus grand détail tous les différents écarts de cette débauche, toutes ses branches, toutes les attenances, ce qu'on appelle en un mot, en langue de libertinage, toutes les passions *.

La bouche du Marquis est tellement proche de la caméra que l'écran se couvre d'une ombre puis devient totalement noir. Sa voix continue pourtant de résonner:

- Voilà, lecteur, l'heure attendue de la vision indicible qui clôt la divine comédie du monde. Le dispositif est prêt pour ton entrée en scène, car c'est ta place et nulle autre qu'il s'agit de commettre. Quiconque s'est avancé au bord de la table de dissection qu'est cette comédie de lecture ne reviendra pas de sa dernière image vidéale. 
En effet, sur la scène cathodique apparaissent finalement les trois corps, noués l'un à l'autre jusqu'à n'en former qu'un seul:

Corps spirant de Dante, vertigineux, incandescent et pleurant, évanouissant et tremblant. Corps de la mémoire rêvant sa langue et se voyant périr à chaque pas.

Corps morcelé de Sade, tailladé, découpé, porté au supplice en sa texture de charnier. Corps débauché, bandant et jutant, s'écoutant pratiquer la boucherie hurlante de sa posture passionnelle.

Corps topologique d'Aquin, sacré dans sa doublure de vidéaste, filmant cette passation inédite de Dante à travers Sade. Corps de la commotion et du regard en proie à sa réversibilité fatale.

Ce corps triple et un n'est-il pas celui de la lecture dont aucune Société des Amis du Crime Télévisé ne saurait se saisir puisqu'il est a-figural? Ce corps gnostique de la passion c'est * vous». Vous, en tant qu'un désir vous pointe à la sortie du texte, au bout du double index de la fiction et de la réalité. Vous, dans le procès anagogique de la lettre, là où se disposent les possibilités concentriques du signifiant.

Mais pourquoi, me direz-vous, l'Enfer et son théâtre? Ce n'était que pour marquer la mise en jeu de toute passion: une physique douloureuse de la lecture en passe de devenir un corps fictif. Je terminerai donc là-dessus :

\section{Chers lecteurs,}

Cette lettre dont j'ai fait ma comédie, je ne l'ai écrite que pour repenser la place du sujet supposé lire. Un sujet que sa position transsémiotique (ou transfinie) garde appendu à l'écriture. Écriture dont l'acte est ici la limite du sujet théorisable ou, si l'on veut, l'enfer de la théorie. En d'autres termes, penser le sujet de la lecture c'est aussi devenir lecteur, c'est-àdire: passionné.

Ce corps passionnel de la lecture, il faudrait l'entendre comme l'atopie du corps parlant qui vient au texte pour accomplir dans le temps ce qui était hors du temps. Enfin ceci : le littéral, la lettre ou la passion du signifiant a lieu chaque fois qu'en ce corps-là, le vôtre, c'est le langage qui dit "je». 